\title{
Préférences ethnobotaniques des espèces ligneuses locales exploitées pour la production d'huile végétale dans le cercle de Sikasso, Mali
}

\author{
Urbain Dembélé (1,2), Yénizié Koné (1), Bino Témé (1), \\ Anne M. Lykke (3), Amadou M. Kouyaté (1) \\ (I) Institut d'Economie Rurale (IER), Mali \\ (2) Institut Supérieur de Formation et de Recherche Appliquée (ISFRA), Mali \\ (3) Department of Bioscience, Aarhus University, Denmark
}

\begin{abstract}
This study examines the preferences of communities in the Mali-South area in terms of local woody species exploited for the production of oil. The study was conducted using ethnobotanical surveys based on semi-structured interviews in two localities of the circle of Sikasso. The surveyed ethnic groups are the Fulani and Senufo in Ziékorodougou, and Bambara and Fulani in Niankorobougou. The sample totaled 48 informants, including twelve defined by ethnic group in both localities. Vitellaria paradoxa, Lophira lanceolata, Carapa procera and Pentadesma butyraceae are the main local tree species identified for the production of oil in the area. Preference ranking scores revealed that $C$. procera is a species of priority importance after $V$. paradoxa. There is a similarity of community preferences in terms of plant species used for the production of edible oil. In contrast, community preference varied in terms of choices for the production of cosmetic oil. This difference appears to be related to the level of knowledge and uses of the products of these plants by local communities. The results of this study will help to integrate the needs of people in term of production of vegetable oil in promotion strategies and sustainable management of forest resources. Key words: indigenous oil trees species, preference ranking, ethnobotany, local knowledge, sub-Saharan Africa
\end{abstract}

Cette étude a mis en évidence les espèces ligneuses locales exploitées pour la production d'huile par les communautés en zone Mali-sud. L'étude a été réalisée à travers des enquêtes ethnobotaniques basées sur des interviews semi-structurées dans deux localités du cercle de Sikasso. Les ethnies enquêtées sont les Sénoufo et les Peulh à Ziékorodougou, les Bambara et les Peulh à Niankorobougou. L'échantillon a totalisé 48 informateurs dont douze par groupe ethnique dans les deux localités. Vitellaria paradoxa, Lophira lanceolata, Carapa procera et Pentadesma butyraceae sont les principales espèces ligneuses locales identifiées pour la production d'huile dans la zone. Les scores de classification préférentielle ont révélé que $C$. procera est une espèce d'importance prioritaire après $V$. paradoxa. Il existe une similitude des préférences des communautés en matière d'espèces végétales exploitées pour la production d'huile alimentaire. Par contre, pour la production d'huile cosmétique une variation des préférences communautaires a été constatée. Cette 
variation semble être liée au niveau de connaissances et d'utilisations des produits de ces plantes par les communautés locales. Les résultats de cette étude vont contribuer à intégrer les besoins des populations en matière de production d'huile végétale dans les stratégies de promotion et de gestion durable des ressources forestières.

Mots clés : arbres oléagineux indigènes, classification préférentielle, ethnobotanique, perceptions locales Afrique subsaharienne

\section{Introduction}

En Afrique subsaharienne, il est largement reconnu que pour assurer leur survie, les populations ont recours aux ressources naturelles, en particulier les ressources forestières. Ces ressources occupent une place de choix dans la vie quotidienne de ces populations grâce aux multiples usages dont elles font l'objet (FAO, 20II). Dans le domaine des ressources forestières, le Mali regorge d'énormes potentialités comme Vitellaria paradoxa, Lannea microcarpa, Balanites aegyptiaca, Sclerocarya birrea dont la valorisation contribuera à l'amélioration de la sécurité alimentaire et à l'accroissement des revenus des populations rurales. De nombreux travaux antérieurs ont montré comment les arbres et les produits forestiers non-ligneux sont utilisés pour l'alimentation humaine (Kouyaté et al. 2009; De Caluwé et al. 20roa; De Caluwé et al. 20rob), la pharmacopée traditionnelle, le fourrage, l'artisanat et l'énergie domestique, (Gustad et al. 2004 ; Havinga et al. 2010; Faye et al. 20I0; Anonymous 20I0). Les travaux de Gakou et al. (I994), Cuny et al. (I999), Sissoko and Kergna (2002), Diop et al. (2005) ont montré que les espèces ligneuses locales présentent une importance tant pour leurs usages naturels que pour leurs valeurs ajoutées à travers la transformation des produits collectés pour répondre à une demande de plus en plus forte sur les marchés nationaux et régionaux.

C'est pourquoi, depuis un certain temps, un intérêt accru est accordé aux recherches sur les espèces ligneuses forestières pour contribuer à leur conservation et gestion durable en vue de multiplier les avantages qu'elles peuvent offrir aux populations. La présente étude vise à documenter les connaissances locales des populations sur les espèces ligneuses oléagineuses locales. Il s'agit d'identifier les espèces ligneuses locales exploitées par les populations pour la production d'huile végétale, déterminer leur préférence en matière d'huile alimentaire et d'huile cosmétique et d'appréhender l'état de conservation de ces ressources ligneuses. Cette étude va servir de base pour l'identification d'espèces oléagineuses locales à promouvoir pour diversifier les sources de revenus et accroitre la sécurité alimentaire des populations. Aussi, la connaissance des préférences des populations locales peut aider à la mise en œuvre de stratégies de gestion durable des ressources naturelles intégrant les spécificités locales (Belem et al. 2007; Camou-Guerrero et al. 2008).

\section{Matériel et méthodes}

Cette étude porte sur les espèces ligneuses locales traditionnellement exploitées par les populations locales pour la production d'huile. 


\section{Sites d'étude}

L'étude a été réalisée en décembre 20II dans deux communes rurales du cercle de Sikasso en zone climatique soudanienne du Mali. Les sites d'étude sont les villages de Niankorobougou dans la commune rurale de Kaboïla et Ziékorodougou dans la commune rurale de Finkolo. Les deux communes font parties du cercle de Sikasso et situées dans la partie Sud du Mali (Figure I). Le climat est de type soudanien et est caractérisé par une alternance entre la saison sèche dominée par des vents secs et la saison des pluies qui durent environ 6 mois. La pluviométrie varie entre IIoo et I400 mm (PIRT, I986). Le domaine forestier couvre plus de $80 \%$ de la superficie totale du cercle et recèle d'importantes ressources. La population du Cercle de Sikasso est essentiellement composée de Sénoufo, Peulh, Gana, Samogo, Bambara, Minianka, Bobo et Dogon.

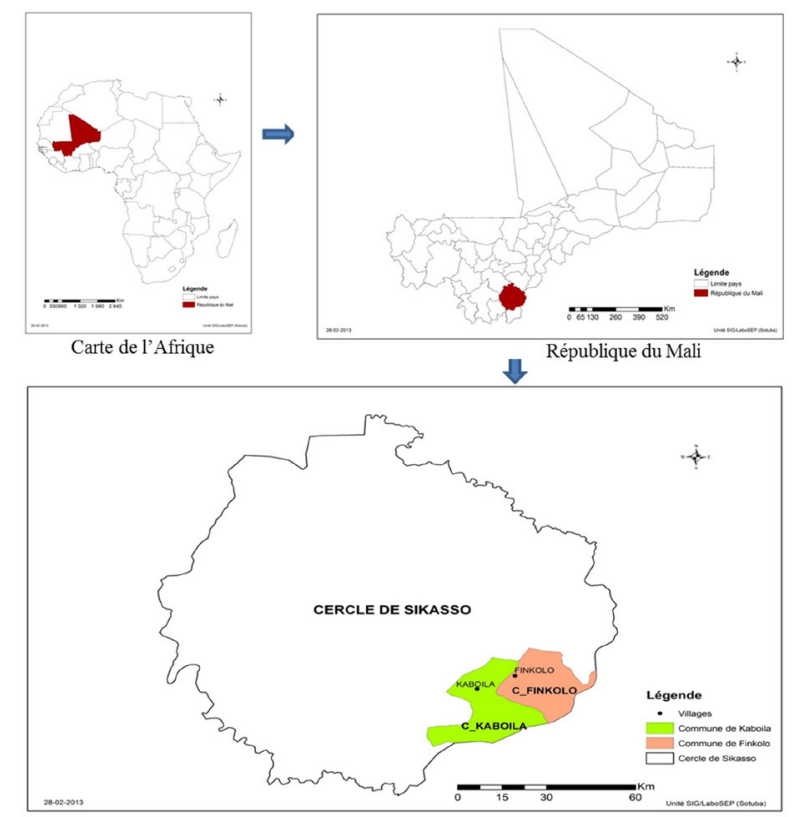

Figure 1 : Localisation de la zone d'étude. 


\section{Méthodes}

Enquêtes ethnobotaniques

La collecte des données a été réalisée en utilisant une approche ethnobotanique. Cette approche est basée sur des enquêtes au niveau des groupes ethniques dans les villages sur la base d'un échantillon stratifié (Lykke, Kristensen, \& Ganaba, 2004). Dans chaque village deux groupes ethniques ont été retenus dont le choix a porté sur le groupe ethnique majoritaire et le groupe ethnique Peulh. L'échantillon d'informateurs était de 6 hommes et 6 femmes au sein de chaque groupe ethnique. Le choix des informateurs a été fait en fonction de leur disponibilité et de leur adhésion aux objectifs de l'étude. Au total 48 informateurs ont été interviewés.

La collecte des données a été réalisée à travers des entretiens individuels sur la base d'un questionnaire semi-structuré. Les questions ont porté sur les espèces ligneuses locales exploitées pour la production d'huile, les préférences pour la production alimentaire, la production cosmétique et de savon et l'état de ces ressources ligneuses dans le terroir. La collecte des données ethnobotaniques a été enrichie par des entretiens et discussions informelles auprès d'autres personnes ressources et à travers les observations de terrain (Martin 2004; Reyes-Garcia et al. 2006).

\section{Traitement et analyses des données}

La méthode d'analyse est adaptée de celle développée par Lawrence et al. (2005) basée sur le calcul d'indicateurs quantitatifs qui, par la suite sont interprétés qualitativement. Chaque informateur a cité par ordre de préférence les espèces ligneuses locales exploitées pour la production d'huile. Par la suite, le rang donné a été converti en un score. Les scores utilisés ont été des notes décroissantes à partir de 5 attribuées à l'espèce suivant l'ordre de citation par l'informateur. Quand une espèce identifiée n'était pas citée par un informateur, la note o était donnée. La classification concernait les cinq premières espèces ligneuses. Pour chaque espèce identifiée, le score moyen a été calculé pour chaque groupe ethnique et selon le sexe.

$$
\mathrm{Vti}=\sum \frac{\mathrm{Ti}}{\mathrm{Ni}}
$$

Vti : le score moyen attribué à une espèce donnée par une catégorie d'informateurs dans une communauté ;

$\mathrm{Ti}$ : la somme des scores donnés à ladite espèce par cette catégorie d'informateurs dans la communauté;

$\mathrm{Ni}$ : le nombre de répondants de cette catégorie d'informateurs de la dite communauté.

Les données collectées ont été traitées et analysées à l'échelle de la zone d'étude, à l'échelle du village et à l'échelle des groupes ethniques et du genre. Le traitement et les analyses des données ont été réalisés à l'aide des logiciels SPSS.I8 (Statistical Package for the Social Sciences) et Excel. Les données ont été analysées en utilisant en premier lieu les indices de la statistique descriptive à travers les distributions de fréquences, les moyennes et les pourcentages. 
Préférences ethnobotaniques des espèces ligneuses locales 


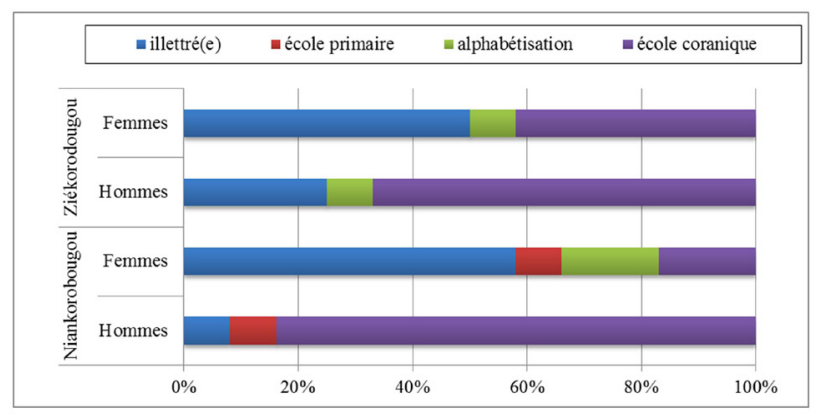

Figure 2 : Niveau d'instruction des informateurs selon le sexe (\%).

Les espèces ligneuses locales exploitées pour la production d'huile

Quatre espèces ligneuses locales ont été identifiées comme étant principalement exploitées pour la production d'huile. Ces espèces identifiées sont Vitellaria paradoxa, Lophira lanceolata, Carapa procera et Pentadesma butyraceae. Elles sont reconnues respectivement sous les appellations de "shii ", " manan ", "kobi » et " waleman » en langue locale Bamanakan.

A Niankorobougou, $67 \%$ des informateurs ont cité en moyenne trois espèces pour la production d'huile, alors qu'à Ziékorodougou ce même pourcentage d'informateurs $(67 \%)$ a cité en moyenne quatre espèces exploitées (Figure 3). Il ressort de cette analyse que les populations à Ziékorodougou ont une meilleure connaissance des espèces oléagineuses qu'à Niankorobougou.

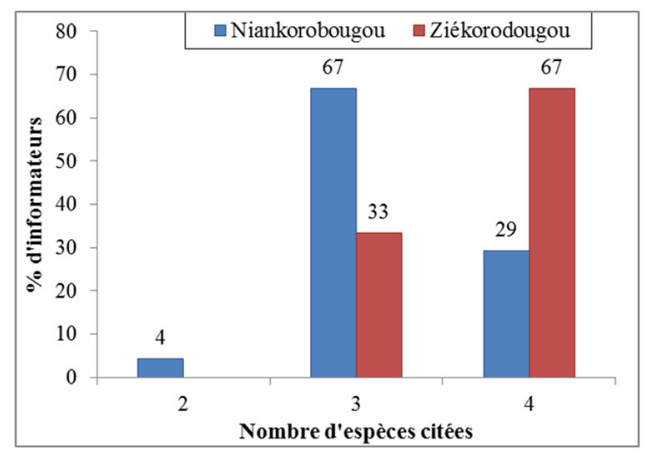

Figure 3 : Taux de citation des espèces exploitées par village. 
Les tableaux 2 et 3 présentent les scores moyens et rang obtenus par espèce.

\begin{tabular}{l|l|l|l|l|l|l|l|l}
\hline \multirow{2}{*}{ Espèces exploitées } & \multicolumn{4}{|c|}{ Niankorobougou } & \multicolumn{3}{c}{ Ziélkorodougou } \\
\hline & \multicolumn{2}{|c|}{ Bambara } & \multicolumn{2}{c|}{ Peulh } & \multicolumn{2}{c}{ Sénoufo } & \multicolumn{2}{c}{ Peulh } \\
\hline & $\begin{array}{l}\text { score } \\
\text { moy. }\end{array}$ & rang & $\begin{array}{l}\text { score } \\
\text { moy. }\end{array}$ & rang & $\begin{array}{l}\text { score } \\
\text { moy. }\end{array}$ & rang & $\begin{array}{l}\text { score } \\
\text { moy. }\end{array}$ & rang \\
\hline Vitellaria paradoxa & 4,92 & I & 5,00 & I & 5,00 & I & 4,92 & I \\
\hline Carapa procera & 2,92 & 3 & 3,08 & 3 & 3,92 & 2 & 4,00 & 2 \\
\hline Lophira lanceolata & 3,83 & 2 & 3,83 & 2 & 2,83 & 3 & 2,75 & 3 \\
\hline Pentadesma butyraceae & 0,58 & 4 & 0,50 & 4 & I,75 & 4 & I,25 & 4
\end{tabular}

Tableau 2 : Score moyen et rang des espèces ligneuses oléagineuses suivant les groupes ethniques.

\begin{tabular}{l|l|l|l|l|l|l|l|l} 
Espèces exploitées & \multicolumn{4}{|c|}{ Niankorobougou } & \multicolumn{4}{c}{ Ziékorodougou } \\
\hline & \multicolumn{2}{|c|}{ Homme } & \multicolumn{2}{c|}{ Femme } & \multicolumn{2}{c}{ Homme } & \multicolumn{2}{c}{ Femme } \\
\hline & $\begin{array}{l}\text { score } \\
\text { moy. }\end{array}$ & rang & $\begin{array}{l}\text { score } \\
\text { moy. }\end{array}$ & rang & $\begin{array}{l}\text { score } \\
\text { moy. }\end{array}$ & rang & $\begin{array}{l}\text { score } \\
\text { moy. }\end{array}$ & rang \\
\hline Vitellaria paradoxa & 5,00 & I & 4,92 & I & 5,00 & I & 4,92 & I \\
\hline Carapa procera & 3,00 & 3 & 3,00 & 3 & 3,83 & 2 & 4,08 & 2 \\
\hline Lophira lanceolata & 3,83 & 2 & 3,83 & 2 & 2,83 & 3 & 2,75 & 3 \\
\hline Pentadesma butyraceae & 0,92 & 4 & 0,17 & 4 & $\mathrm{I}, 25$ & 4 & $\mathrm{I}, 75$ & 4
\end{tabular}

Tableau 3 : Score moyen et rang des espèces ligneuses oléagineuses suivant le sexe.

Les résultats au tableau 2 montrent que $V$. paradoxa obtient le score moyen le plus élevé au niveau de tous les groupes ethniques dans les deux villages et demeure la première espèce préférée. A Niankorobougou la deuxième place est attribuée à L. lanceolata suivi en troisième position par C. procera. Par contre à Ziékorodougou, la seconde place est revenue à $C$. procera et L. lanceolata se classe au troisième rang. La quatrième place est occupée par P. butyraceae qui recueille la plus faible valeur au niveau de tous les groupes ethniques. Les résultats selon le sexe au tableau 3 suivent la même tendance. Ces résultats montrent qu'à l'échelle du village, les préférences pour les espèces ligneuses exploitées sont les mêmes quel que soit le groupe ethnique et le genre. Par conséquent, on peut affirmer que les communautés à l'échelle du village ont relativement les mêmes connaissances sur les espèces ligneuses exploitées dans leur terroir. Les valeurs de classification des espèces montrent que $V$. paradoxa est la première espèce préférée quel que soit le groupe ethnique et le genre. Ce rang de première place obtenu par V. paradoxa a été aussi rapporté par de nombreux auteurs (Diop et al. 2005 ; Belem et al. 2008). Des travaux plus récents ont également identifié $V$. paradoxa comme une espèce caractéristique de la composante ligneuse 
des systèmes agroforestiers et à forte indice d'utilisation totale (Anonymous 2010 ; Guigma et al. 2012). Ces résultats confirment l'importance de cette espèce ligneuse pour les populations par de nombreux auteurs. Faye et al. (2010) ont noté que les fruits de V. paradoxa (Photo ra) sont largement consommés à l'état cru pour leur pulpe par les populations dans la région de Ségou pendant la période soudure qui correspond à la saison des pluies. Ambé (200I) a également mentionné que V. paradoxa est exploité pour ses fruits par les Malinké en Côte d'Ivoire. En plus de V. paradoxa, les autres produits exploités pour la production d'huile par les populations locales sont les graines de L. lanceolata (Photo Ib), les noix de C. procera (Photo Ic) et de P. butyraceae (Photo Id). Dans ses travaux, Ambé (200I) a également indiqué que $C$. procera et $P$. butyraceae étaient des fruitiers exploités pour l'extraction d'huile par le groupe socioculturel Malinké en Côte d'Ivoire.

Les scores de classification préférentielle obtenus à l'échelle de village montrent que L. lanceolata occupe le second rang à Niankorobougou alors qu'à Ziékorodougou c'est $C$. procera qui arrive à la seconde place (Tableau 4). Toutefois, à l'échelle régionale (échelle de la zone d'étude), les scores moyens de classification obtenus donnent les préférences suivantes par ordre de priorité : V paradoxa, C. procera, L. lanceolata et P. butyraceae. $C$. procera apparait comme une espèce d'importance capitale après $V$. paradoxa. Ce résultat s'accorde avec les travaux d'autres auteurs qui ont montré que $C$. procera est une espèce qui mérite une attention particulière dans la zone Nord-guinéenne du Mali (FAO I996 ; Maiga 200I).

\begin{tabular}{l|l|l|l|l|l|l} 
Espèces exploitées & \multicolumn{2}{|c|}{ Niankorobougou } & \multicolumn{2}{c|}{ Ziékorodougou } & \multicolumn{2}{c}{ Régional } \\
\hline & score moy. & rang & score moy. & rang & score moy. & rang \\
\hline Vitellaria paradoxa & 4,96 & I & 4,96 & I & 4,96 & I \\
\hline Carapa procera & 3,00 & 3 & 3,96 & 2 & 3,48 & 2 \\
\hline Lophira lanceolata & 3,83 & 2 & 2,79 & 3 & $3,3 \mathrm{I}$ & 3 \\
\hline Pentadesma butyraceae & 0,54 & 4 & I,50 & 4 & I,02 & 4
\end{tabular}

Tableau 4 : Score moyen et rang des espèces ligneuses oléagineuses à l'échelle du village et au niveau régional. 


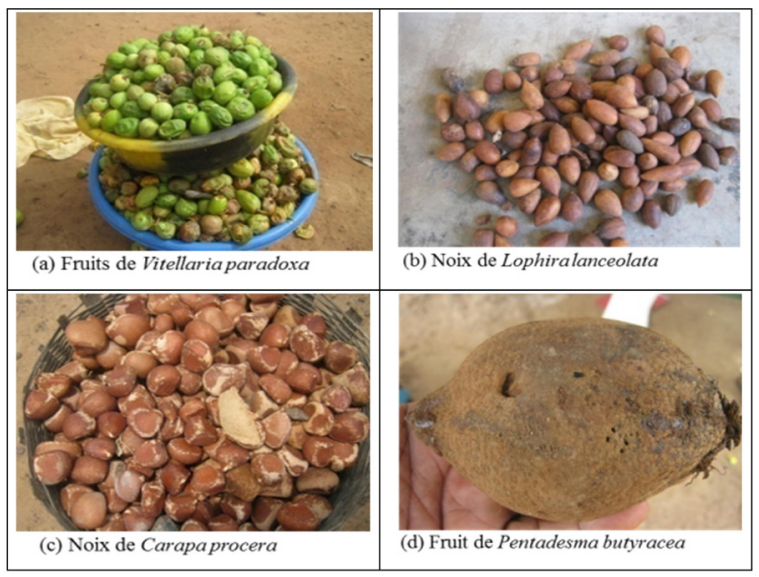

Photo 1 : Produits exploités des espèces ligneuses pour la production d'huile.

Préférences des populations pour la production d'huile alimentaire

En ce qui concerne les préférences des communautés pour la production d'huile alimentaire, trois espèces ont été retenues. Il s'agit de V. paradoxa, L. lanceolata et P. butyraceae dont les huiles sont utilisées pour les besoins alimentaires par les populations.

Les scores de classification préférentielle des espèces pour la production alimentaire suivant les groupes ethniques et le genre (Figures 4 et 5) suivent la même tendance dans les deux villages. Ce résultat suggère qu'il existe donc une similitude des préférences des populations dans les deux localités en matière d'espèces alimentaires. 


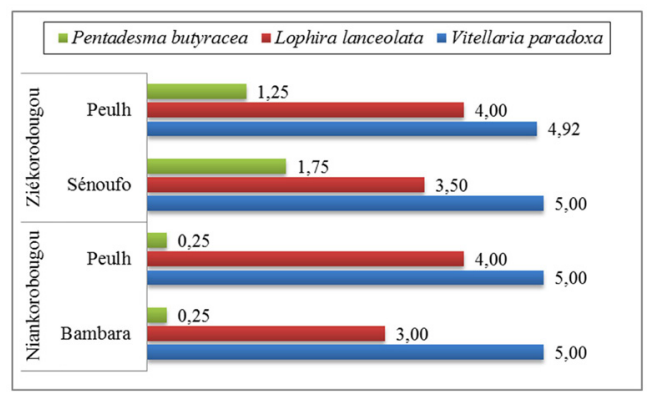

Figure 4 : Score moyen de classification préférentielle des espèces pour la production d'huile alimentaire suivant les groupes ethniques.

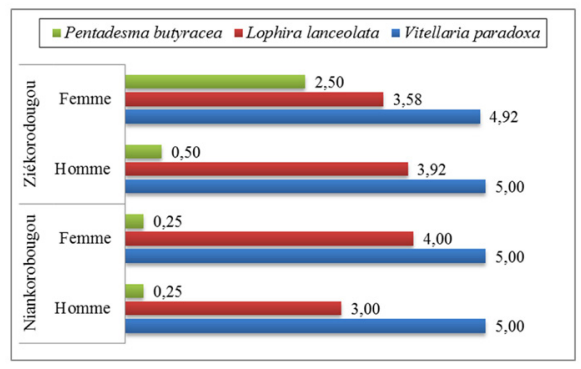

Figure 5 : Score moyen de classification préférentielle des espèces pour la production d'huile alimentaire suivant le genre.

V. paradoxa est la première espèce préférée pour l'usage alimentaire de son huile au niveau des groupes ethniques ainsi qu'au niveau du genre (Figures 4 et 5 ). Son beurre (Photo 2a) demeure depuis des siècles la principale source d'huile végétale pour les populations locales. Il est reconnu qu'en milieu rural, le beurre de V. paradoxa, ou plus communément appelé beurre de karité, joue un rôle primordial dans l'alimentation et constitue une importante source de revenus en particulier pour les femmes qui en sont les principales exploitantes. Ce rôle est confirmé dans les travaux de Faye et al. (2010) qui ont rapporté la grande valeur attribuée au beurre de V. paradoxa par les femmes. Dans des travaux antérieurs, Diarassouba et al. (2008) ont également rapporté une forte consommation du beurre de V. paradoxa chez les Sénoufo de la Côte d'Ivoire. Boffa (I999) dans ses travaux a estimé à Io kg par personne et par an la consommation moyenne de beurre de karité en Afrique sub-saharienne. Pouliot (2012) a trouvé que les revenus issus de la consommation et de la commercialisation des fruits, des noix et du beurre de V. paradoxa représentaient une contribution moyenne de $7 \%$ du revenu total des ménages au Burkina Faso. 
L. lanceolata est la deuxième espèce exploitée pour la production d'huile alimentaire. L'huile extraite de ses graines (Photo 2c), est utilisée dans l'alimentation par les populations qui l'assimilent à l'huile d'arachide pour sa ressemblance. Cependant, les populations ont expliqué que la production de l'huile de L. lanceolata était faible. Cette faible production s'explique dans une certaine mesure par la pénibilité de son extraction et ses graines plus dures et plus petites que celles de V. paradoxa et qui nécessitent plus de temps de collecte pour amasser une quantité appréciable de noix. L. lanceolata est parfois confondu avec V. paradoxa, d'où son nom de faux-karité ou "shii sina » en langue locale Bamanakan. Toutefois, dans les deux sites les populations accordent un grand intérêt pour L. lanceolata.

La troisième espèce exploitée par les populations pour la production d'huile alimentaire est P. butyraceae. Le beurre extrait des amandes de cette espèce ligneuse est aussi consommée par les populations. Les utilisations du beurre de P. butyraceae ont été rapportées par certains auteurs. Les travaux de Natta et al. (2010) ont signalé l'utilisation alimentaire du beurre de P. butyraceae par des groupes socioculturels au Nord-Ouest du Bénin. Comparable au beurre de V. paradoxa, le beurre de P. butyraceae est moins collant et a l'odeur moins prononcée que celui-ci. Du fait de sa couleur, il est souvent appelé beurre de karité jaune ou beurre de karité doré (Photo 2b). P. butyraceae a obtenu un score de classification plus élevée à Ziékorodougou qu'à Niankorobougou. Dans ce dernier village, les hommes et les femmes ont exprimé un même niveau de préférence pour P. butyraceae pour la production d'huile alimentaire alors qu'à Ziékorodougou le score attribué par les femmes est plus élevé que celui des hommes (Figure 5).

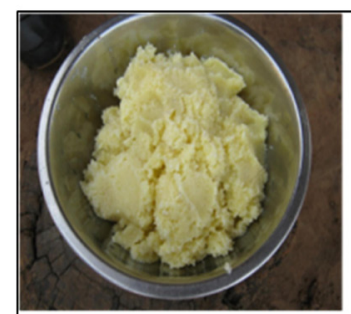

(a) Beurre de karité

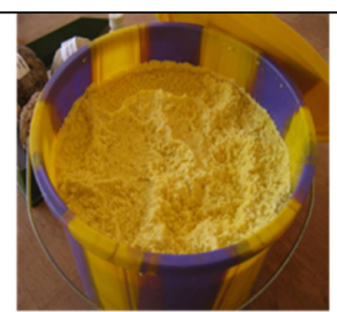

(b) Beurre de Pentadesma butyracea

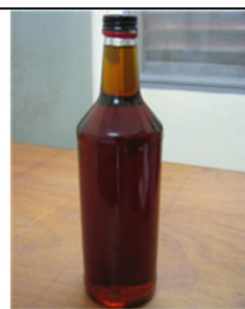

(c) Huile de Lophira lanceolata

Photo 2 : Beurres et huile végétales utilisées pour l'alimentation humaine.

Préférences pour la production d'huile cosmétique et de savon

Pour la production d'huile cosmétique et de savon, quatre espèces ont été identifiées. Ce sont respectivement L. lanceolata, $C$. procera et $V$. paradoxa et $P$. butyraceae. 


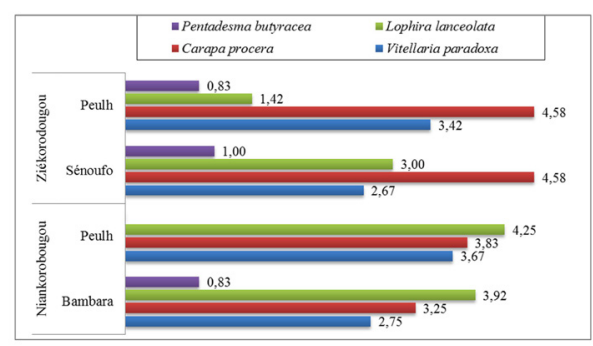

Figure 6: Score moyen de classification préférentielle des espèces pour la production d'huile cosmétique et de savon suivant les groupes ethniques.

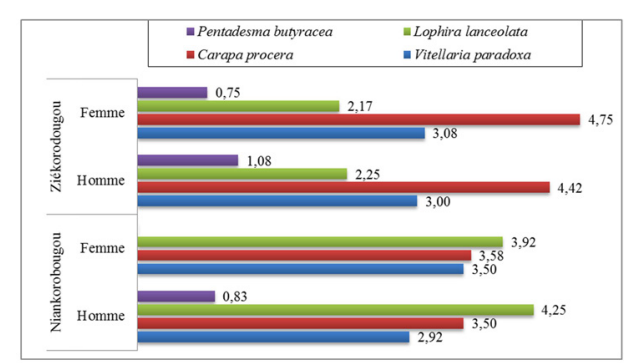

Figure 7 : Score moyen de classification préférentielle des espèces pour la production d'huile cosmétique et de savon suivant le genre.

Pour la production d'huile cosmétique et de savon, les résultats ont montré une variation des préférences entre les sites et suivant les groupes ethniques (Figure 6). A Ziékorodougou, les Sénoufo et les Peulh ont une même préférence pour C. procera qui a obtenu le plus fort score de classification $(4,58)$. L. lanceolata occupe le second rang de préférence $(3,00)$ chez les Sénoufo alors que les Peulh ont accordé leur préférence pour le second rang à V. paradoxa $(3,42)$. Par contre, à Niankorobougou, L. lanceolata est la première espèce préférée pour la production cosmétique et de savon par les Bambara et les Peulh. Les autres préférences sont similaires dans ce village pour les deux ethnies sauf dans le cas de P. butyraceae qui n'est pas cité par les Peulh.

L'analyse suivant le genre a montré que les hommes et les femmes à Ziékorodougou ont les mêmes préférences pour la production cosmétique et de savon. Suivant les scores de classification préférentielle obtenus, $C$. procera occupe le premier rang suivi de V. paradoxa et en troisième position L. lanceolata (Figure 7). A Niankorobougou les femmes et les hommes ont également les mêmes préférences pour la production cosmétique et de savon. Les scores de classification accordent la première préférence à L. lanceolata suivi en deuxième position par $C$. procera et au troisième rang $V$. paradoxa.

Ces résultats montrent que le beurre de karité est davantage préféré pour les besoins alimentaires que pour la production cosmétique et de savon. La variation des préférences pour la production cosmétique et de savon peut s'expliquer par le niveau de 
connaissances et d'utilisations des produits de ces plantes par les communautés locales. Ce constat semble être justifié par le fait que les populations ne consomment pas l'huile de $C$. procera à cause de son goût amer et par conséquent, le préfèrent pour la production cosmétique et d'autres usages. Ces résultats sont similaires aux travaux de Boffa (I999) qui a rapporté que l'utilisation des produits des espèces agroforestières varie suivant les groupes ethniques selon les goûts et la disponibilité. Les utilisations de l'huile de C. procera ont été rapportées par de nombreux auteurs. Guèye et al. (2009) ont montré que l'huile de Carapa était principalement utilisée en médicine pour le traitement de certaines maladies, en cosmétique et pour la fabrication de savon. Weber et al. (2010) ont rapporté également que l'huile de Carapa est utilisée comme savon, huile de massage, lotion insecticide et autres utilisations cosmétiques.

\section{Etat des ressources ligneuses locales}

Les résultats ont montré que le mode de gestion actuel des droits de propriétés et d'usages sur les arbres des parcs agroforestiers en général et des espèces oléagineuses en particulier a favorisé un maintien de l'état des ressources ligneuses locales selon $58 \%$ et 7I \% des informateurs respectivement à Niankorobougou et à Ziékorodougou (Figure 8). Cependant, une certaine dégradation de l'état des ressources ligneuses locales est observée selon $25 \%$ des informateurs à Ziékorodougou et $17 \%$ à Niankorobougou. Cette tendance de dégradation semble se justifier à travers les observations de terrain relevées à travers l'exploitation et la vente de bois de chauffe à Ziékorodougou qui constitue en effet un site d'approvisionnement pour la ville de Sikasso. Mais d'une façon générale, les populations expliquent la dégradation des ressources ligneuses locales par la déforestation à travers les défrichements de nouveaux champs de culture du fait de la culture extensive et de l'augmentation du nombre d'exploitations agricoles. Cependant, la pratique la plus courante de gestion et de conservation des espèces ligneuses oléagineuses est la préservation des arbres dans les champs de culture. V. paradoxa est cité en exemple comme la première espèce protégée dans ce cadre. En effet, les espèces de la composante arborée sont épargnées lors des défrichements pour la mise en culture à cause de leur rôle socio-économique très important pour les populations rurales (Anonymous, 2010). En dehors de la régénération naturelle les communautés n'ont pas développé de moyen spécifique de conservation pour les autres espèces hors champ comme $C$. procera, L. lanceolata et P. butyraceae. 


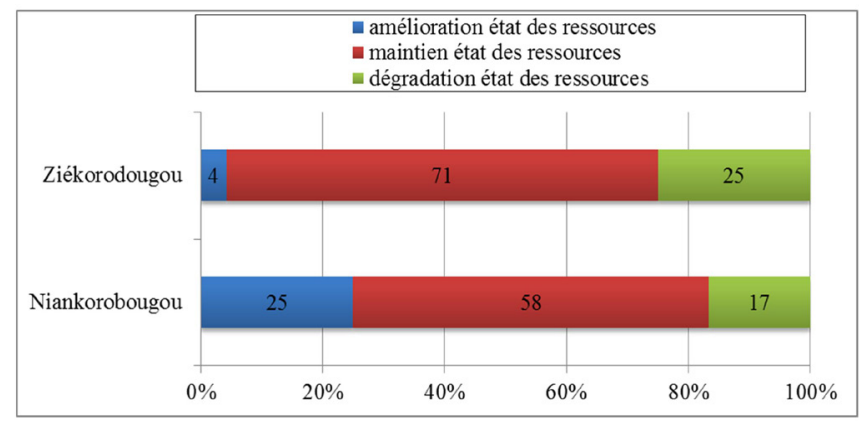

Figure 8: Etat des ressources ligneuses locales basé sur les connaissances locales.

Facteurs influençant l'exploitation des produits d'une espèce ligneuse

L'impact de valorisation des produits forestiers non ligneux a été évalué à travers les facteurs qui influencent l'exploitation d'une espèce ligneuse locale. Trois facteurs essentiels ont été recensés comme influençant l'exploitation d'une espèce ligneuse. Il s'agit de l'intérêt alimentaire, de l'intérêt économique pour la génération de revenus et de l'intérêt thérapeutique. Il ressort de ces facteurs que l'intérêt alimentaire est le plus important. L'intérêt alimentaire a recueilli $50 \%$ et $54 \%$ de citations respectivement à Niankorobougou et à Ziékorodougou (Figure 9). Ces résultats corroborent des travaux antérieurs qui ont mis en exergue l'importance alimentaire et la contribution des espèces ligneuses indigènes à la sécurité alimentaire des populations (Buchmann et al. 20I0; De Caluwé et al. 20ıo; De Caluwé et al. 20Iob). A Niankorobougou, l'intérêt économique a totalisé $40 \%$ de citations alors que l'intérêt thérapeutique totalisait io \%. Comparativement à Ziékorodougou, ces deux facteurs ont enregistré des taux de citations similaires de $22 \%$ et $24 \%$ respectivement pour l'intérêt thérapeutique et l'intérêt économique (Figure 9). Il ressort de ces résultats que l'intérêt économique constitue le second facteur suivi en troisième position par l'intérêt thérapeutique. Ces résultats obtenus sont différents de ceux rapportés par Faye et al. (2010) qui ont trouvé que l'intérêt thérapeutique était le critère le plus important suivi en seconde position par l'intérêt alimentaire et en troisième place par l'intérêt économique pour la génération de revenus. Niang et al. (2005) ont fait également ressortir que les critères les plus importants pour lesquels un arbre est apprécié et donc entretenu dans le parc agroforestier sont sa capacité de fournir, entre autres, nourriture, produits médicinaux, bois de chauffe et fourrage.

C'est pourquoi dans leurs travaux, Gustad et al. (2004) ont proposé que les études sur les produits forestiers non ligneux nécessitent différentes méthodes adaptées à chaque situation. Ils ont rapporté que les utilisations des produits forestiers non ligneux sont sujettes aux changements saisonniers et que les produits sont distribués différemment au cours de l'année. 


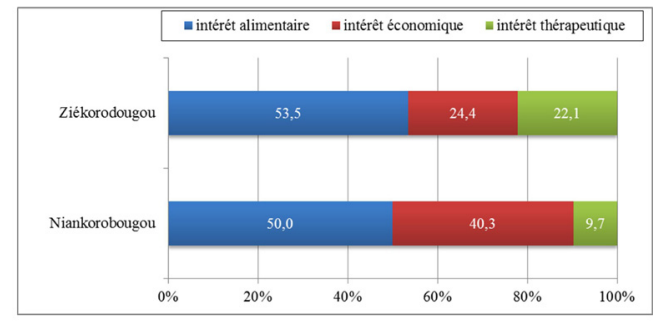

Figure 9: Facteurs influençant l'exploitation des produits d'une espèce ligneuse.

\section{Conclusion}

Cette étude a mis en évidence les espèces ligneuses naturelles exploitées par les populations dans deux communes rurales au Mali pour la production d'huile. Ces espèces sont Vitellaria paradoxa, Lophira lanceolata, Carapa procera et Pentadesma butyraceae.

Les résultats de l'étude ont permis de confirmer l'importance particulière accordée à $V$. paradoxa par les populations. Les résultats ont également montré l'intérêt particulier que les espèces L. lanceolata, $C$. procera et $P$. butyraceae jouent dans la vie socioéconomique des populations. L'huile de L. lanceolata est autant préférée que celle de V. paradoxa pour l'alimentation humaine par les communautés. Par contre, l'huile de $C$. procera a été identifiée comme utilisée principalement pour la production cosmétique et de savon à cause de son goût amer.

Ces résultats montrent que les connaissances locales des populations sur les espèces ligneuses exploitées sont indispensables pour mieux appréhender les potentialités de valorisation de ces espèces. Ces connaissances locales sont également essentielles pour comprendre les pratiques de gestion locales qui soutiennent ces espèces ligneuses. Les résultats vont contribuer à intégrer les besoins des populations en matière de production d'huile végétale dans les stratégies de promotion et de gestion durable des ressources ligneuses locales.

En dehors de la régénération naturelle les communautés n'ont pas développé de moyen spécifique de conservation pour les espèces hors-champ comme C. procera, L. lanceolata et P. butyraceae. C'est pourquoi, il serait nécessaire de développer des stratégies de domestication de ces espèces afin de pouvoir préserver la biodiversité et soutenir leurs potentialités de valorisation.

\section{Remerciements}

Les auteurs remercient DANIDA (Danish International Development Agency) pour le financement du projet QualiTree (Danida Io-002AU) et les populations des villages enquêtés pour leur contribution à cette recherche. 


\section{Références}

Ambé, G.-A. (200I). Les fruits sauvages comestibles des savanes guinéennes de Côte-d'Ivoire: état de la connaissance par une population locale, les Malinké. Biotechnologie Agronomie Société et Environnement, 5(I), $43-58$.

Anonymous. (2010). Caractérisation des Systèmes Agroforestiers de la Région de Ségou. Etude bibliographique. FUNCITREE Report no.I. 20I0. Bamako, February 2010.

Belem, B., Nacoulma, B.M.I., Gbangou, R., Kambou, S., Hansen, H.H., Gausset, Q., Boussim, I.J. (2007). Use of Non Wood Forest Products by local people bordering the "Parc National Kaboré Tambi", Burkina Faso. Te Journal of Transdisciplinary Environmental Studies, 6(I), I-2I.

Belem, B., Olsen, C.S., Theilade, I., Bellefontaine, R., Guinko, S., Lykke, A.M., ... Boussim, J. I. (2008). Identification des arbres hors forêt préférés des populations du Sanmatenga (Burkina Faso). Bois et Forêts Des Tropiques, 298(4), 53-64.

Boffa, J.M. (1999). Agroforestry Parklands in Sub-Saharan Africa. FAO Conservation Guides. FAO Conservation Guides. Rome: Food and Agricultural Organization of the United Nations.

Buchmann, C., Prehsler, S., Hartl, A., \& Vogl, C.R. (2010). The importance of baobab (Adansonia digitata L.) in rural West African subsistence - suggestion of a cautionary approach to international market export of baobab fruits. Ecology of Food and Nutrition, 49(3), I45-172.

Camou-Guerrero, A., Reyes-Garcia, V., Martinez-Ramos, M., \& Casas, A. (2008). Knowledge and Use Value of Plant Species in a Raràmuri Community: A Gender Perspective for Conservation. Human Ecology, 36(2), 259-272.

Cuny, P., Sanogo, S., \& Nadine, S. (I999). Arbres du domaine soudanien. Leurs usages et leur multiplication. Sikasso, Mali: Institut d'Economie Rurale - Intercoopération Suisse.

De Caluwé, E., Halamová, K., \& Van Damme, P. (20roa). Adansonia digitata L. - A review of traditional uses, phytochemistry and pharmacology. Africa Focus, 23(I), II-5I.

(20Iob). Tamarindus indica L. - A review of traditional uses, phytochemistry and pharmacology. Africa Focus, $23(\mathrm{I}), 53-83$.

Diarassouba, N., Koffi, K. E., N'Guessan, K. A., Van Damme, P., \& Sangare, A. (2008). Connaissances locales et leur utilisation dans la gestion des parcs à karité en Côte d'Ivoire. Africa Focus, 2I(I), 77-96.

Diop, M., Kaya, B., Niang, A., \& Olivier, A. (2005). Les espèces ligneuses et leurs usages: les préférences des paysans dans le Cercle de Ségou au Mali. (No. n 9 ).

FAO. (1996). Arbres du terroir pour l'alimentation. Projet GCP/RAF/303/ITA, Forêt et sécurité alimentaire en Afrique Sahélienne, Sikasso.

(20II). Les forêts au service de la nutrition et de la sécurité alimentaire. Rome, Italie.

Faye, M.D., Weber, J.C., Mounkoro, B., \& Dakouo, J.-M. (20ro). Contribution of parkland trees to farmers' livelihoods: a case study from Mali. Development in Practice, 20(3), 428-434.

Gakou, M., Force, J. E., \& MClaughlin, W. J. (I994). Non-timber forest products in rural Mali: a study of villager use. Agroforestry Systems, 28(I), 213-226.

Guèye, M., Kenfack, D., \& Forget, P.-M. (2009). Importance socio-culturelle, potentialités économiques et thérapeutiques du Carapa (Meliaceae) au Sénégal. In X. van der Burgt, J. van der Maesen \& J.-M. Onana (eds), Systematics and conservation of African plants. (pp. 357-365). Royal Botanic Gardens, Kew.

Guigma, Y., Zerbo, P., Millogo-Rasolodimby, J., G.Y., Zerbo, P., \& Millogo-Rasolodimby, J. (2012). Utilisation des espèces spontanées dans trois villages contigus du Sud du Burkina Faso. Tropicultura, 30(4), 230-235.

Gustad, G., Dhillion, S. S., \& Sidibé, D. (2004). Local use and cultural and economic value of products from trees in the parklands of the municipality of Cinzana, Mali. Economic Botany, 58(4), 578-587.

Havinga, R.M., Hartl, A., Putscher, J., Prehsler, S., Buchmann, C., \& Vogl, C. R. (2010). Tamarindus indica L. (Fabaceae): patterns of use in traditional African medicine. Journal of Ethnopharmacology, I27(3), 573588. http://doi.org/ro.Ior6/j.jep.2009.II.028

Kouyaté, A.M., Van Damme, P., De Meulenaer, B., \& Diawara, H. (2009). Contribution des produits de cueillette dans l'alimentation humaine. Cas de Detarium microcarpum. Africa Focus, 22(I), 77-88. 
Lawrence, A., Phillips, O.L., Ismodes, A.R., Lopez, M., Rose, S., Wood, D., \& Farfan, A.J. (2005). Local values for harvested forest plants in Madre de Dios, Peru : towards a more contextualised interpretation of quantitative ethnobotanical data. Biodiversity and Conservation, I4, 45-79.

Lykke, A.M., Kristensen, M.K., \& Ganaba, S. (2004). Valuation of local use and dynamics of 56 woody species in the Sahel. Biodiversity and Conservation. I3(Io), I96I-I9go.

Maiga, A.S. (200I). Situation des ressources génétiques forestières du Mali. In Atelier sous-régional FAO/IPGRI/ CIRAF sur la conservation, la gestion, l'utilisation durable et la mise en valeur des ressources génétiques forestières de la zone sahélienne. Ouagadougou, 22-24 sept. 1998.

Martin, G.J. (2004). Ethnobotany. A methods manuel. (Earthscan, Ed.). London. New York: Earthscan.

Natta, A., Sogbégnon, R., \& Tchobo, F. (2010). Connaissances Endogènes et Importance du Pentadesma butyracea (Clusiaceae) pour les Populations Autochtones au Nord Ouest Bénin. Fruit, Vegetable and Cereal Science and Biotechnology, 4, I8-25.

Niang, A., Kaya, B., Kindt, R., Rouxel, C., Galleti, S., Mounkoro, B., ... Garneau, C. (2005). Quantification of village level agroforestry biodiversity according to management unit and its livelihood functions in three villages of the Ségou region, Mali.

PIRT. (I986). Zonage agro-écologique du Mali. Commission consultative nationale d'évaluation et de suivi de la mise en cuvre de la stratégie alimentaire. Groupe zonage écologique et adéquation des projets (p. 15I). Projet Inventaire des Ressources Terrestres.

Pouliot, M. (2012). Contribution of "Women's Gold" to West African Livelihoods : The Case of Shea (Vitellaria paradoxa) in Burkina Faso. Economic Botany, 66(3), 237-248.

Reyes-Garcia, V., Huanca, T., Vadez, V., Leonard, W., \& Wilkie, D. (2006). Cultural, practical and economic value of wild plants : a quantitative study in the Bolivian Amazon. Economic Botany, 6o(I), 62-74.

Sissoko, P., \& Kergna, A. O. (2002). Etude diagnostique des circuits de commercialisation des produits du jujubier dans la zone sahélienne de Mali: cas de l'arrondissement de Kayes. In Second Regional Workshop on Socioeconomic Aspects of Agroforestry in the Sahel. (pp. 76-82). Bamako, Mali. 4-6 mars.

Weber, N., Birnbaum, P., Forget, P.-M., Guèye, M., \& Kenfack, D. (2010). L'huile de carapa (Carapa spp., Meliaceae) en Afrique de l'Ouest: utilisations et implications dans la conservation des peuplements naturels. Fruits, 65(6), 343-354. http://doi.org/I0.105I/fruits/2010029 